\title{
Environmental equalizer for underwater communications
}

\author{
António Silva and Sérgio M. Jesus \\ Institute for Systems and Robotics, Universidade do Algarve \\ Campus de Gambelas, PT-8005-139 Faro, Portugal \\ Email: \{asilva,sjesus\}@ualg.pt \\ João Gomes \\ Institute for Systems and Robotics, Instituto Superior Técnico \\ Av. Rovisco Pais, PT-1049-001 Lisboa, Portugal \\ Email:.jpg@isr.ist.utl.pt
}

\begin{abstract}
This paper presents an environmental-based equalization algorithm for underwater communications. This algorithm is based on the passive time-reversal (pTR) and waveguide invariant properties of ocean channels. Passive time-reversal allows for the implementation of a simple communications system, but it loses performance in the presence of geometric mismatch between the probe-signal and the actual data symbols transmission. The waveguide invariant properties state that geometric mismatches, both in depth and range, can be partially compensated by applying an appropriate frequency shift in the passive time-reversal operator. Results with binary PSK data at a carrier frequency of $3.6 \mathrm{kHz}$, collected during the MREA'04 sea trial, show that the Mean Square Error (MSE) between the transmitted and the received data symbols remains stable at least to a range mismatch of about $37.5 \mathrm{~m}$ in the presence of source depth and an array depth oscillations of approximately $0.7 \mathrm{~m}$. In such conditions, when comparing the proposed pTR equalizer with plain pTR, an overall gain of approximately $4.11 \mathrm{~dB}$ in output MSE is achieved.
\end{abstract}

\section{INTRODUCTION}

In recent years Time Reversal (TR) has received particular attention from the scientific community. After practical demonstration of its spatial-temporal focusing capabilities in the ocean [1] several applications of active TR (aTR), from tomography to communications, were suggested [1], [2]. Passive TR uses a receive-only array, and a probe-signal is transmitted ahead of the data for channel Impulse Response (IR) estimation. The IR estimate is then used as a synthetic channel for temporal focusing of the data signal, which is equivalent to the deconvolution of the multipath generated by the real channel.

When applied to underwater coherent communications the achieved TR focus is not perfect due to errors on the IR estimate and the time variability of the channel, resulting in uncompensated intersymbol interference (ISI) [3]. That problem is even more relevant in communications with a moving source and/or receiver. In that case it is intuitive that a rapid degradation of passive TR temporal focusing will occur due to the increasing mismatch between the assumed and actual channels. In order to guarantee a longer stability of the focal spot, three solutions are usually proposed: one is to transmit probe-signals more frequently; another is to use an adaptive algorithm to track the IR from the initial probe signal IR estimation; and finally a third alternative is to use a low-complexity equalizer with only one coefficient per channel. A performance comparison between those adaptive pTR variants is presented in [4]. The major inconvenient of the first method is that frequent transmission of probe-signals reduces the overall transmission rate. In the second the channel estimates are represented by a large number of coefficients that have to be adapted. The third case seems to be the best compromise between complexity and efficiency. In this paper a different approach is proposed by considering that the environment mismatch between the probe-signal and data transmission is mainly due to the geometric properties (source-array range, source depth and array depth), and that the knowledge of such mismatch can be used to compensate for the overall pTR IR mismatch between the probe-signal and actual data transmissions.

A physics-based algorithm for pTR equalization based on the waveguide invariant properties of the shallow water channel is proposed. The waveguide invariant property $\beta$ [5] has been applied to change the aTR range focus in [6], and to interpret a model for performance prediction of a time-reversal communication system [7]. In [8] a new waveguide invariant $\zeta$ was identified, which, in conjunction with $\beta$, allows for the compensation of pTR geometric mismatch. Though this waveguide invariants, changes on geometric features of the acoustic channel, such as source-receiver range, source depth and array depth, can be compensated by a frequency shift in the estimate IRs during the probe-signal transmission. The resulting Frequency Shift pTR (FSpTR) equalizer will increase the pTR output power, resulting in lower MSE. A physics-based equalizer for underwater communications that is able to detect the transmitted data sequence and to simultaneously estimate the source-array range, source depth and array depth is thus obtained.

The reliability of the physics-based waveguide invariant pTR equalizer is demonstrated using experimental data obtained during the MREA'04 sea trial, where binary PSK signals at a data rate of 400 bits per second were transmitted with a carrier 
frequency of $3.6 \mathrm{kHz}$. Results obtained with FSpTR, after Doppler compensation of the received signals, show a long-term compensation of channel mismatch, with the MSE remaining stable up to a source-array range mismatch of about $37.5 \mathrm{~m}$ in the presence of source depth variations between $71.6 \mathrm{~m}$ and $72.3 \mathrm{~m}$ and an array depth oscillation of approximately 0.63 $\mathrm{m}$. In such conditions, when comparing FSpTR with plain pTR, an overall gain of approximately $4.11 \mathrm{~dB}$ in output MSE is achieved.

The paper is organized as follows: Section II recalls the use of pTR in underwater communications; in Section III the pTR geometric mismatch compensation using frequency shifts is presented; Section IV develops the FSpTR equalizer, which is applied to real data in section V; Section VI summarizes the main results, draws some conclusions, and suggests future research.

\section{Passive Time Reversal apPlied to communications}

The basic setup for applying pTR to communications consists in a point source that sends information to a Vertical Line Array (VLA). The procedure starts by sending a short probe-signal, waiting for the channel to clear of multipath arrivals, and then sending the data stream. Considering the noise less case, the received probe and the data are processed in a TR fashion and it results that the pTR output, in its baseband version [9], is given by

$$
y(t)=\left[a(t) * p_{4}(t)\right] * p_{T R}(t, \Delta),
$$

where the lower $*$ represents the convolution, $\left[a(t) * p_{4}(t)\right]$ is the transmitted signal with raised-cosine pulse shape symbols; $p_{T R}(t, \Delta)$ is the resulting pTR IR acoustic field given by

$$
p_{T R}(t, \Delta)=\sum_{i=1}^{I} h_{i}(t, \Delta) * h_{i}^{\prime *}(-t),
$$

where and the upper $*$ represents phase conjugation, $i$ is the hydrophones index, $h_{i}^{\prime}(t)$ represents the channel IRs during probe transmission, $h_{i}(t, \Delta)$ is the channel IRs during data transmission, and $\Delta$ represents a possible mismatch between $h_{i}^{\prime}$ and $h_{i}$.

In a shallow water waveguide and at a range greater than a few water depths the acoustic field generated by a monochromatic point source at the $i^{\text {th }}$ hydrophone of a VLA is given by the so-called Green's function

$$
G_{\omega}\left(r, z_{0}, z_{i}\right)=\frac{-j}{\rho \sqrt{8 \pi R}} e^{-j \frac{\pi}{4}} \sum_{n=1}^{M} \frac{Z_{m}\left(z_{i}\right) Z_{m}\left(z_{0}\right)}{\sqrt{k_{m}}} e^{j k_{m} R}
$$

where $m$ is the mode number, $M$ is the total number of propagating modes, $\rho$ is the (constant) water density, $R$ is the source-array range, $Z_{m}$ is the $m^{t h}$ mode shape, $z_{0}$ is the source depth, $z_{i}$ is the $i$-hydrophone depth, and $k_{m}$ is the $m^{t h}$ mode horizontal wavenumber. In a range independent environment and under the adiabatic condition $k_{m}$ and $M$ are the only quantities in (3) that depend on frequency $\omega$. In the frequency domain the synthetic pTR IR acoustic field (2) in a stationary environment (considering $\Delta$ to be negligible) is given by [10]

$$
\begin{aligned}
P_{p c}\left(R, z_{0}, z_{i}, \omega\right) & =\sum_{i=1}^{I} G_{\omega}\left(R, z_{0}, z_{i}\right) G_{\omega}^{*}\left(R, z_{0}, z_{i}\right) \\
& =\frac{1}{\rho^{2} 8 \pi R} \sum_{m=1}^{M} \sum_{n=1}^{M} \frac{Z_{m}\left(z_{0}\right) Z_{n}\left(z_{0}\right)}{\sqrt{k_{m} k_{n}}} \sum_{i=1}^{I} Z_{m}\left(z_{i}\right) Z_{n}\left(z_{i}\right) e^{j\left(k_{m} R-k_{n} R\right)} \\
& =\frac{1}{\rho^{2} 8 \pi R} \sum_{m=1}^{M} \frac{\left|Z_{m}\left(z_{0}\right)\right|^{2}}{\left|k_{m}\right|}
\end{aligned}
$$

where, for simplicity, it was considered that $Z_{m}(\cdot)$ and $k_{m}$ are real quantities, which amounts to ignoring the leaky modes and considering the loss mechanisms to be negligible [11]. Additionally, in (4) it was considered that the array spans the entire water column and that hydrophones depth sampling is sufficiently dense to fulfil the modal orthogonality property.

Since $k_{m}$ in the denominator of (4) is a weak function of frequency, $P_{p c}(\cdot)$ is approximately constant. Thus in time domain the $p_{T R}(\cdot)$ given by (2) can be approximated by a pulse under convolution with the bandlimited transmitted sequence.

\section{PASSIVE TIME REVERSAL GEOMETRIC MiSMATCH COMPENSATION}

When there is a geometric mismatch $\Delta$ ( $\Delta r$ for source-array range, $\Delta z_{0}$ for source depth, $\Delta z_{i}$ for array depth, or any combination of those) between the probe and data transmissions it can be shown [8] that the mismatch in one of them can be 
compensated by applying a frequency shift $\Delta \omega$ to the other, and (4) becomes

$$
\begin{aligned}
P_{p c}\left(\cdot, \Delta \omega ; \Delta r, \Delta z_{0}, \Delta z_{i}\right)= & \sum_{i}^{I} G_{\omega}\left(R+\Delta r, z_{0}+\Delta z_{0}, z_{i}+\Delta z_{i}\right) G_{\omega+\Delta \omega}^{*}\left(R, z_{0}, z_{i}\right) \\
= & \frac{1}{\rho^{2} 8 \pi R} \sum_{m=1}^{M} \sum_{n=1}^{M} \frac{Z_{m}\left(z_{0}\right) Z_{n}\left(z_{0}+\Delta z_{0}\right)}{\sqrt{k_{m} k_{n}}} \sum Z_{m}\left(z_{i}\right) Z_{n}\left(z_{i}+\Delta z_{i}\right) \\
& e^{j\left(k_{m}^{\dagger} R-k_{n}(R+\Delta r)\right)},
\end{aligned}
$$

where $k_{m}^{\dagger}=k_{m}(\omega+\Delta \omega)$, the influence of the frequency shift on the horizontal wavenumber $k_{m}$ placed in the denominator of (5) is neglected and the range shift is considered to be small enough so that $R \approx \sqrt{R(R+\Delta r)}$ in the denominator. In (5) it is relevant that the source-array range shift $\Delta r$ perturbs the exponential term, the array depth shift $\Delta z_{i}$ perturbs the modes orthogonality and the source depth shift $\Delta z_{0}$ perturbs the gain that is related with the focal spot depth in aTR (see [8] for details). A full discussion of this perturbation mechanism is presented in [8] and [12]. From this discussion it turns out that the frequency shift $\Delta \omega$ used in (5) can be computed as

$$
\Delta \omega=\frac{\omega}{R}\left(-\Delta r \beta+\Delta z_{i} \zeta_{i}+\Delta z_{0} \zeta_{0}\right)
$$

and is applied in (5) by considering the first order Taylor expansion

$$
k_{m}(\omega+\Delta \omega) \approx k_{m}(\omega)+\frac{d k_{m}(\omega)}{d \omega} \Delta \omega,
$$

where the derivative of $k_{m}$ with respect to $\omega$ represents the horizontal group slowness. In (6) $\beta, \zeta_{i}$ and $\zeta_{0}$ are the waveguide invariants related to $\Delta r, \Delta z_{i}$ and $\Delta z_{0}$, respectively. Since $\zeta_{i}$ and $\zeta_{0}$ have a similar nature, in the following $\zeta$ will be used to represent both.

The invariant $\beta$ is a well-known invariant in the frequency/range plane [5] and is selected from a set of values

$$
\beta_{m n}=\frac{\frac{1}{v_{h, n}}-\frac{1}{v_{h, m}}}{\frac{1}{u_{h, n}}-\frac{1}{u_{h, m}}},
$$

where $v_{h, m}=\omega / k_{m}$ is the horizontal phase velocity and $u_{h, m}$ is the horizontal group velocity of mode $m$. The selection of $\beta$ from the $\beta_{m n}$ set must take in consideration the best linear approximation in the least-squares sense of the horizontal wavenumber $k_{m}$ using the horizontal group slowness for a limited number of of modes $M_{e}<M$, that is,

$$
k_{m} \approx-\frac{d k_{m}}{d \omega} \omega \beta+\omega \rho \quad \beta,
$$

where $\beta$ represents the slope of the approximation and $\rho_{\beta}$ its offset. The invariant $\rho_{\beta}$ is closely related with $\beta$ and is selected in a set of values

$$
\rho_{\beta, m n}=\frac{1}{v_{h, m}}+\beta_{m n} \frac{1}{u_{h, m}} .
$$

With the approximation of $k_{m}$ (9) the compensation of the range mismatch is straightforward since the range mismatch $\Delta r$ and the frequency shift compensation $\Delta_{\omega}$ given by (6) both affect (5) in the exponential term. Such compensation can be applied to narrowband signals since $\beta$ and $\rho_{\beta}$ are invariant with frequency, in a similar manner to the focal intensity range shift for aTR proposed in [6].

The $\zeta$ waveguide invariants used for source depth $\Delta z_{0}$ and array depth $\Delta z_{i}$ compensation have been developed in [8] in a similar manner to $\beta$, but for the approximation of the vertical wavenumber $\gamma_{m}$ using the horizontal group slowness

$$
\gamma_{m} \approx-\frac{d k_{m}}{d \omega} \omega \zeta+\omega \rho \quad \zeta
$$

$\zeta$ is selected from a set of values

$$
\zeta_{m n}=\frac{\frac{1}{v_{v, n}}-\frac{1}{v_{v, m}}}{\frac{1}{u_{h, n}}-\frac{1}{u_{h, m}}}
$$

where $v_{v, m}=\omega / \gamma_{m}$ is the vertical phase velocity, and $\rho_{\zeta}$ is selected in the set

$$
\rho_{\zeta, m n}=\frac{1}{v_{v, m}}+\zeta_{m n} \frac{1}{u_{h, m}} .
$$


The application of (11) in (5) for the source depth mismatch $\Delta z_{0}$ and array depth mismatch $\Delta z_{i}$ compensation was made possible after establishing that they can be partially compensated using $\exp \left(j \gamma_{m} \Delta z_{0}\right)$ and $\exp \left(j \gamma_{m} \Delta z_{i}\right)$ respectively (see [8] for details). That is, for source depth mismatch $\Delta z_{0}$ compensation

$$
\sum_{m=1}^{M} Z_{m}\left(z_{0}\right) Z_{m}\left(z_{0}+\Delta z_{0}\right) e^{j \gamma_{m} \Delta z_{0}} \approx \sum_{m=1}^{M} Z_{m}\left(z_{0}\right) Z_{m}\left(z_{0}\right) \frac{V\left(\Delta z_{0}\right)}{2},
$$

where $\left|V\left(\Delta z_{0}\right)\right| \approx 2$ for small values of $\Delta z_{0}$ and $\left|V\left(\Delta z_{0}\right)\right| \approx 1$ for higher values of $\Delta z_{0}$. For the array depth mismatch $\Delta z_{i}$ compensation

$$
\sum_{i=1}^{I} Z_{m}\left(z_{i}\right) Z_{n}\left(z_{i}+\Delta z_{i}\right) e^{j \gamma_{m} \Delta z_{i}} \approx \sum_{i=1}^{I} Z_{m}\left(z_{i}\right) Z_{n}\left(z_{i}\right) \frac{W\left(m, \Delta z_{i}\right)}{2},
$$

where $\left|W\left(m, \Delta z_{i}\right)\right| \approx 2$ for small values of $\Delta z_{i}$ and $\left|W\left(m, \Delta z_{i}\right)\right|$ oscillates around 1 for higher values of $\Delta z_{i}$.

With the approximation of $\gamma_{m}$ (11) and the compensation mechanisms (14) and (15) the compensation of the source and array depth mismatches is straightforward since the mismatch can be compensated by a complex exponential of $\gamma_{m}$ and the frequency shift compensation $\Delta \omega$, given by (6), affects (5) in an exponential factor. Such compensation can be applied to narrowband signals since $\zeta$ and $\rho_{\zeta}$ are invariant with frequency.

As previously suggested for the invariant $\beta$, the $\zeta$ compensation mechanism has a better performance when considering an effective number of modes $M_{e}<M$. The selection of $\beta$ in (8) and $\zeta$ in (12) for optimal compensation depends on $M_{e}$. Such dependence suggests that the compensation mechanism can be further optimized by considering its application to smaller groups of modes instead of a single group of modes from $m=1, . ., M_{e}$, resulting in different values of $\beta$ and $\zeta$ for each group. That was previously proposed in [6] and [13] for the waveguide invariant $\beta$.

In a waveguide with a depth-dependent sound-speed profile the vertical wavenumber as well as phase velocity become depth dependent. In the present paper it is assumed that such dependence is small enough so that $\zeta_{i}$ can be calculated from the mean phase velocity, $\overline{v_{v, m}\left(z_{i}\right)}$, over the array. Since $\zeta_{0}$ is computed for the nominal source depth $z_{0}$ in a real situation it will be expected that $\zeta_{i} \neq \zeta_{0}$.

\section{THE PASSIVE TIME-REVERSAL FREQUENCY SHIFT EQUALIZER}

Figure 1 shows the pTR system adapted to incorporate a frequency shift that compensates for the geometric mismatch $\Delta$ between the IR for probe-signal transmission $h^{\prime}(t)$ and the IR during data transmission $h(t)$. It behaves as a matched filter (to the IR) demodulator with a set of $L$ frequency shifts being applied to the channel IR $h^{\prime}(t)$ estimate after time windowing, where the optimal frequency shift is selected based on the "Maximum power selection" block with the $z_{l}(t)$ power being computed in time slots. In figure 1 the pTR output signal can be written as

$$
\mathrm{z}_{l}(t)=y_{l}(t)+x 1_{l}(t)+x 2_{l}(t)+x 3_{l}(t),
$$

where index $l$ designates frequency shift $\Delta \omega_{l}, y_{l}(t)$ contains the desired data-signal contaminated with ISI and the other three terms are noise disturbances (see [9] for definitions and details).

The overall Signal to Noise Ratio (SNR) ia a ratio between the variance of the signal term $y_{l}(t)$ and a sum of the variances of the noise disturbances $x 1_{l}(t), x 2_{l}(t)$ and $x 3_{l}(t)$. It can be shown that the effect of the frequency shift compensation on the channel IR $h^{\prime}(t)$ estimate is higher on the signal variance term than on the noise variances, therefore contributing to SNR enhancement. Therefore, tracking the maximum power of the pTR output $\mathrm{z}_{l}(t)$ gives a clear indicator of the best frequency shift taking into account the actual environmental/geometric conditions of the signals being received. That results in the FSpTR system that equalize the real pTR IR $\sum_{i=1}^{I} h_{i}(t) * h_{i, t_{0}, \tau}^{\prime *}(-t)$ (where the ' indicates that there is a geometric mismatch $\Delta$ between $h_{i}$ and $h_{i, t_{0}, \tau}^{\prime *}$ ) with the non geometric mismatch pTR IR $\sum_{i=1}^{I} h_{i}(t) * h_{i, t_{0}, \tau}^{*}(-t)$, where $t_{0}$ and $\tau$ are the starting time and duration of the time window respectively.

\section{ReAl Data Application}

The experimental data were acquired during the MREA'04 sea trial that took place off the town of Setúbal, approximately $50 \mathrm{~km}$ south of Lisbon (Portugal), in April 2004. The pTR experiment started at a close range of $0.6 \mathrm{~km}$ to the south of the receiving array (in a gently up sloping region) and the source progressively opened range to the southeast up to $2 \mathrm{~km}$ (with a progressive slope reduction up to range independence). The environment was characterized by a water column depth ranging between 90 to $110 \mathrm{~m}$ over a $1.5 \mathrm{~m}$ silt bottom and gravel layer. The receiving array was free drifting in a surface suspended Acoustic Oceanographic Buoy (AOB) [14], which was found to oscillate vertically due to the surface waves with amplitude of approximately $0.63 \mathrm{~m}$ and frequency between 0.43 and $0.4 \mathrm{~Hz}$, as measured by a wave rider buoy placed in the area of the experiment. The acoustic source was suspended from the NRV Alliance, its depth was measured at a sampling rate of $1 \mathrm{~s}$ with a $10 \mathrm{~cm}$ resolution depth sensor. In the data set processed in this paper it oscillates with a main component of $0.1 \mathrm{~Hz}$, between 71.64 and $72.24 \mathrm{~m}$. 


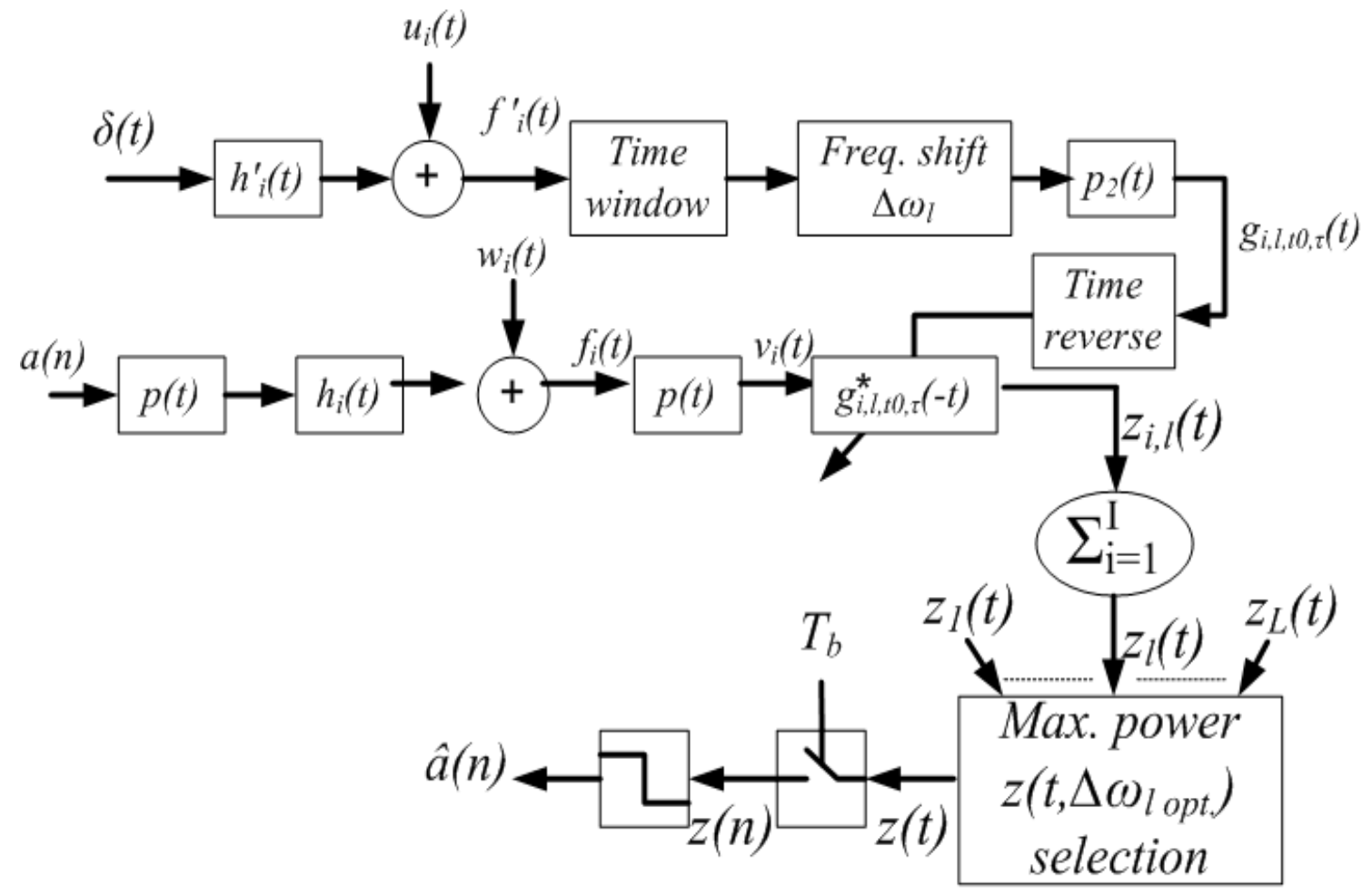

Fig. 1. Block-diagram of the FSpTR equalizer. The blokes path above represent the probe-signal IRs estimate, time windowing, and frequency shift operations. The blokes path middle represents the data transmission and crosscorrelation with the IRs estimate obtained path above, the summation over the $I$ hydrophones gives $L$ pTR processor outputs $\mathrm{z}_{l}(t)$. The blokes path below represents the selection of $\mathrm{z}_{l}(t)$ with the frequency shift that best compensates for the geometric mismatch between probe and data transmissions, and the transmitted symbols estimation.

During the MREA'04 sea trial the pTR based data communications conceptual system was similar to that of figure 1, with the $p_{2}(t)$ narrowband filter of the IR estimation operation (path above in figure 1) distributed between the transmitter and the receiver, i.e., the transmitted probe-signal was a fourth-root raised-cosine pulse and IR estimates were obtained by correlating the received probe-signal with the transmitted one (see [3] for details).

This paper analyzes modulated data at a carrier frequency of $3.6 \mathrm{kHz}$, using a symbol rate of 400 baud and 2-PSK constellation. Fourth-root raised cosine signaling pulses with 100\% roll-off were used such that the signal bandwidth is 800 $\mathrm{Hz}$. Each individual transmission comprises a single truncated PAM signaling pulse acting as a channel probe with symmetric guard intervals for a total duration of $1 \mathrm{~s}$, followed by a $20 \mathrm{~s}$ data packet. The source sequentially transmits 4 packets for a total duration of $84 \mathrm{~s}$. In order to demonstrate the long-term stability of the proposed FSpTR compensation mechanism only the third channel probe pulse was used in the pTR operation of figure 1 to estimate the transmitted symbols in the four data packets. The data was preprocessed using the Doppler compensation method as proposed in [4].

Since the sound speed profile is composed of a thermocline of approximately $20 \mathrm{~m}$ and $1512 \mathrm{~m} / \mathrm{s}$ over a down refracting sound speed up to approximately $1505 \mathrm{~m} / \mathrm{s}$ near the bottom two different modal structures have been observed by the top two hydrophones and by the six bottom hydrophones [13] [8]. Those two modal structures are expected to have different invariants [13], and for the purpose of showing the usefulness of the waveguide invariant frequency shift in underwater communications with pTR in a geometric variable environment, only the six hydrophones below the thermocline were considered.

The system of figure 1 requires the use of a time window whose optimum length $\tau_{o p t}$, in a non geometric mismatch case, has been found in [9]. The time window initial point $t_{0}$ is set to an arbitrary point before the first arrival and the time window length $\tau$ is set to a value higher that the optimum in order to allow for a better behavior of the compensation mechanism in presence of geometric mismatch. In the ray mode analogy [15] later arrivals are related with higher modes, making the time window operate as a mode filter.

By considering the same frequency shift $\Delta \omega_{l}$ to be applied to all modes captured in the time window $\left(t_{0}, \tau\right)$ in the system of figure 1 the surface of figure 2 results. The surface represents the pTR output power $\sigma_{z}^{2}(t)$, computed in time slots of 0.5 $\mathrm{s}$, as a function of time and frequency shift and the blank spaces represent the the elapsed time between the data slots. The solid line traces the maximum of the surface as a function of time, and the '*' indicates the time instant where the channel probe used in the pTR processor was received. GPS data, acquired during the experiment, shows that during the $80 \mathrm{~s}$ of data transmission the source-array range increases by about $50 \mathrm{~m}$, which can be clearly seen in figure 2 as a mean decrement of optimal frequency shift from about $150 \mathrm{~Hz}$ to about $-50 \mathrm{~Hz}$ with $0 \mathrm{~Hz}$ at the channel probe location. Oscillations around the 
mean increment seem to be mainly due to the other geometric mismatches (array depth and source depth), but no definitive conclusions can be draw since there is no ground truth information about array or source depth variations.

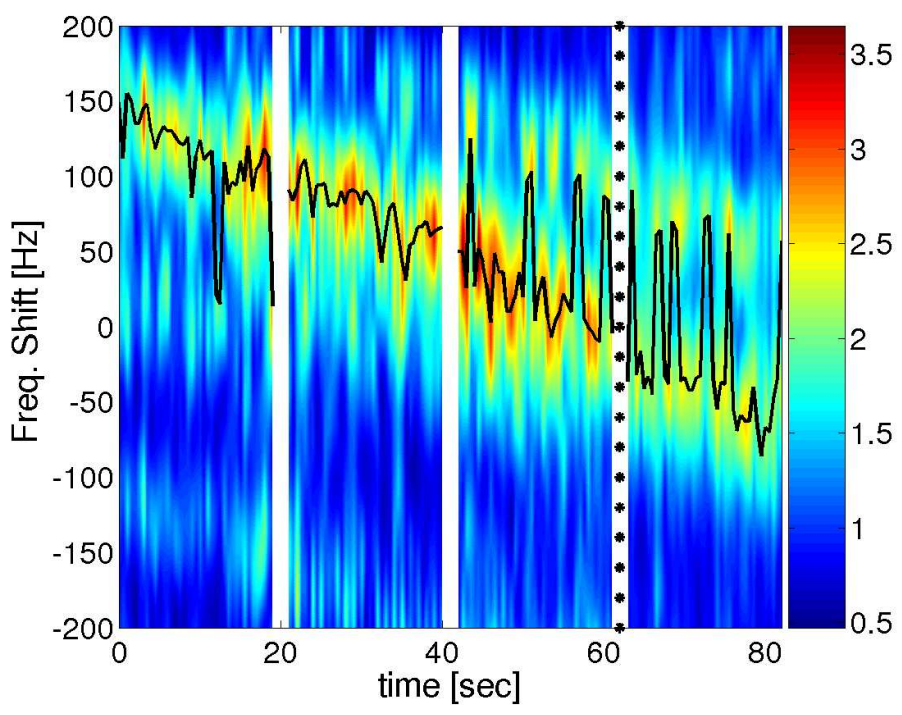

Fig. 2. Passive time-reversal output mean magnitude $\mathbf{z}_{l}(t)$ as a function of time and applied frequency shift, computed by slots of $0.5 \mathrm{~s}$. The solid line traces the maximum of the surface.

Figure 3 shows the MSE between the estimated and the transmitted bit sequence, computed in slots of 0.5 seconds, for plain pTR with no compensation (solid line) and for pTR with compensation (red dashed line). The solid line initially shows a strong degradation in the uncompensated pTR that progressively reduces when approaching time of $t=60 \mathrm{~s}$. Compensated results (red dashed line) maintain the MSE at approximately the same level, resulting in a strong gain. In the first $20 \mathrm{~s}$ that gain is about $5.55 \mathrm{~dB}$, in the second $20 \mathrm{~s}$ it is about $5.7 \mathrm{~dB}$, in the third $20 \mathrm{~s}$ is about it $1.64 \mathrm{~dB}$ and in last $20 \mathrm{~s}$ it is about $1.53 \mathrm{~dB}$, for an overall gain of about $4.11 \mathrm{~dB}$. It is remarkable that such results were obtained with a range mismatch up to about $37.5 \mathrm{~m}$ with source depth varying between $71.6 \mathrm{~m}$ and $72.3 \mathrm{~m}$ and an array depth oscillation of approximately $0.63 \mathrm{~m}$.

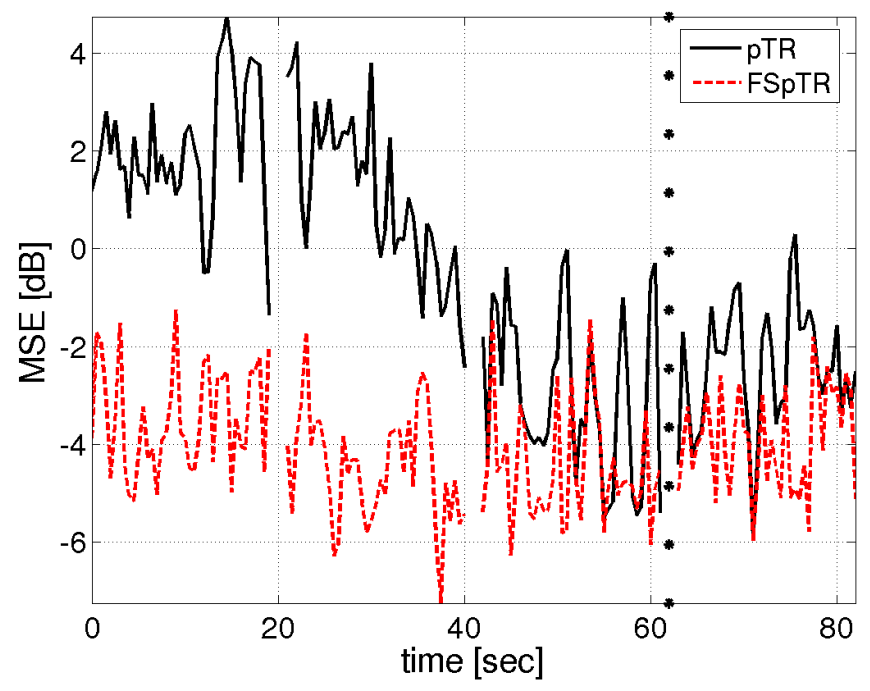

Fig. 3. Mean squared error between the estimated and transmitted data symbols for plain pTR (black line) and FSpTR (red dashed line).

\section{CONClusions And FUture WORK}

Experimental results were given for time-reversed demodulation of $3.6 \mathrm{kHz}$ binary PSK data collected during the MREA'04 experiment. Two receiving architectures were compared: plain pTR and pTR with frequency shift for geometric mismatch compensation - FSpTR. By itself, pTR suffers a significant performance penalty due to the geometric mismatch during the data 
transmission resulting in acceptable results only a $20 \mathrm{~s}$ window around the channel probe location. FSpTR presents a longer stability that was shown to be effective for up to $60 \mathrm{~s}$ from the channel probe location. Moreover, FSpTR presents a gain of $1.5 \mathrm{~dB}$ over pTR even for short-term mismatch. Future work should address a comparison between FSpTR and other adaptive pTR systems [4].

Due to its environmental based nature, FSpTR can be used not only to attain reliable underwater communications, but also to track the geometric variations during data transmission considering a previous estimation of the waveguide invariants $\beta$, $\zeta_{i}$ and $\zeta_{0}$. Future experiments should be planed in order to explore such possibility.

In the present paper the Doppler compensation has been performed off-line previously to the equalization process. Future work should address its inclusion in the equalizer processor. In what concerns symbol synchronization, an heuristic method that chooses the minimal spread of the constellation previously to the slicer operation (slot by slot), was adopted.

\section{ACKNOWLEDGMENTS}

This work was supported by Fundação para a Ciência e a Tecnologia under programs POCI, POCTI and POSI. The authors would like to thank the NATO Undersea Research Centre for the organization of MREA'04 sea trial, NRV Alliance master and personnel and the scientist in charge Emanuel F. Coelho.

\section{REFERENCES}

[1] W.A. Kuperman, W. Hodgkiss, H. Chun Song, T. Akal, C. Ferla, and D. Jackson. Phase conjugation in the ocean: Experimental demonstration of an acoustic time-reversal mirror. J. Acoust. Soc. Am., 103(1):25-40, January 1998.

[2] R.D. Jackson and R.D. Dowling. Phase conjugation in underwater acoustics. J. Acoust. Soc. Am., 89(1):171-181, January 1991.

[3] A. Silva and S. Jesus. Underwater communications using virtual time-reversal in a variable geometry channel. In Proc. MTS/IEEE Oceans'2002, pages 2416-2421, Biloxi, USA, November, 2002.

[4] J. Gomes, A. Silva, and S.M. Jesus. Spatial combining for passive time-reversed communications. submitted J. Acoust. Soc. America, March 2007.

[5] G. A. Grachev. Theory of acoustic field invariants in layered waveguide. Acoust. Phys., (39):33-35, 1993.

[6] H. C. Song, W. A. Kuperman, and W. S. Hodgkiss. Time-reversal mirror with variable range focusing. J. Acoust. Soc. Am., 103((6)):3234-3240, June 1998.

[7] D. Rouseff. Intersymbol interference in underwater acoustic communications using time-reversal signal processing. J. Acoust. Soc. America, 117(2):780 $788,2005$.

[8] A. Silva, S.M. Jesus, and J. Gomes. Generalization of waveguide invariants and application to passive time reversal. submitted to J. Acoust. Soc. America, July 2007.

[9] A. Silva, S.M. Jesus, and J. Gomes. Time reversal optimization for underwater communications. Submitted J. Acoust. Soc. America, April 2007.

[10] D. Rouseff, D.R. Jackson, W.L.J. Fox, D.C. Jones, J.A. Ritcey, and D.R. Dowling. Underwater acoustic communications by passive-phase conjugation: Theory and experimental results. J. Oceanic Engineering, 26(4):821-831, 2001.

[11] F. Jensen, W. Kuperman, M. Porter, and H. Schmidt. Computational Ocean Acoustics. AIP Series in Modern Acoustics and Signal Processing, New York, 1994.

[12] A. Silva, S. Jesus, and J. Gomes. Depth and range shift compensation using waveguide invariant properties. In Proc. of the UAM'07, Heraklion, Crete, Greece, June 2007.

[13] W.A. Kuperman, S. Kim, G.F. Edelmann, W.S. Hodgkiss, H.C. Song, and T. Akal T. Group and phase speed analysis for predicting and mitigating the effects of fluctuations. In Jensen Pace, editor, Impact of Littoral Environment Variability on Acoustic Predictions and Sonar Performance, pages 279-286, Kluwer, September 2002.

[14] A. Silva, F. Zabel, and C. Martins. Acoustic oceanographic buoy telemetry system. Sea Technology, 47(9), September 2006.

[15] I. Tolstoy and C.S. Clay. Ocean Acoustics: Theory and experiments in underwater sound. AIP, New York, 1966. 